\title{
Structure of an RNA Silencing Complex in CRISPR-mediated Immunity
}

\author{
Michael S. Spilman ${ }^{1}$, Alexis I. Cocozaki ${ }^{1}$, Yaming Shao ${ }^{1}$, Nancy F. Ramia ${ }^{1}$, Hong Li ${ }^{1}{ }^{2}$, and Scott \\ Stagg ${ }^{1,2}$ \\ 1. Institute of Molecular Biophysics, Florida State University, Tallahassee, FL 32306, USA. \\ 2. Department of Chemistry and Biochemistry, Florida State University, Tallahassee, FL 32306, USA.
}

Bacteria and archaea employ invader-derived DNA captured in CRISPR (Clustered Regularly Interspaced Short Palindromic Repeat) loci to prevent further infection. The invader-derived DNA are transcribed and processed to short RNAs (crRNA) that are incorporated into effector ribonucleoprotein particles for guided destruction of foreign nucleic acids. The Pyrococcus furiosus $\mathrm{Cmr}$ complex is a Type III, multi-component RNA-cleaving effector that includes six CRISPR-associated proteins (Cmr16) and a target-recognition RNA derived from the CRISPR locus [1]. With no requirement of additional factors, the Cmr complex cleaves RNA complementary to crRNA [1]. Type I effector complexes, exemplified by the Cascade complex of Escherichia coli, and type II, such as those of Streptococcus thermophilus and Streptococcus pyogenes, target DNA [2]. The apparent differences among the three types of effector complexes highlight the need for detailed structural studies of these complexes.

We utilized random conical tilt coupled with single-particle refinement to generate an initial, lowresolution reconstruction of negatively stained $\mathrm{Cmr}$ complexes. High-throughput cryo-electron microscopy analysis was used to reconstruct an intact $\mathrm{Cmr}$ complex bound with its target RNA at $\sim 13 \AA$. Assignment of protein subunits in the Cmr complex was possible from 3D reconstructions of negatively stained Cmr complexes lacking specific subunits and crystal structure fitting. The Cmr complex comprises a helical repeat of two subunits that is capped on both ends by the four remaining subunits. The 'foot' region of the particle contains Cmr2 and Cmr3. Emanating from the Cmr2-Cmr3 foot as a heterodimer and stacking upwards in a right-handed twist are three Cmr4-Cmr5 repeats, which have similar helical features to those observed in isolated $\mathrm{Cmr} 4-\mathrm{Cmr} 5$. Directly above the Cmr4-Cmr5 repeats is Cmr6 that is then followed by Cmr1. We identified the location of the target RNA by calculating a difference map between Cmr complexes with and without target. Difference peaks were observed at the tip, in a central cavity and along the helical back of the complex. The difference peak at the tip of the particle is proximal to but not overlapping the density corresponding to Cmr1, suggesting a colocalization of Cmrl and part of the target RNA.

This architecture is surprisingly similar at the level of subunit organization to the Type I E. coli Cascade complex, which also exhibits a helical arrangement with central repeating units [3]. Similarly, crRNA binds along the helical body with its 5'-handle anchored at the foot region of the complex. This structural similarity between effector complexes of different functions (targeting RNA vs. DNA) suggests a possibility of shared functionalities. Additionally, the structural organization suggests previously unknown insights on possible sites of RNA cleavage. Previous structural and biochemical studies eliminated the both $\mathrm{Cmr} 2$ and $\mathrm{Cmr} 3$ subunits from being the catalytic subunits [4,5]. Thus, the subunits that could possibly catalyze the RNA cleavage reaction are Cmr1, Cmr4, Cmr5 and Cmr6. Among these, Cmr1, Cmr4 and Cmr6 belong to the RAMP family of proteins that include crRNAprocessing endoribonucleases. Locations of these subunits are consistent with the primary cleavage site in the target RNA. Identification of the catalytic subunits and their interactions with the RNA duplex can now be focused on these four subunits. 
References:

[1] C Hale et al, Cell 139 (2009), p. 945-56.

[2] D Bhaya, M Davison and R Barrangou, Annu Rev Genet 45 (2011), p. 273-97.

[3] B Wiedenheft et al, Nature 477 (2011), p. 486-9.

[4] A Cocozaki et al, Cell 20 (2012), p.545-53.

[5] Y Shao et al, Cell (2012), doi: 10.1016/j.str.2013.01.002

[6] The authors acknowledge funding from the National Institutes of Health, grant 1R01 GM099604-01 to H.L.
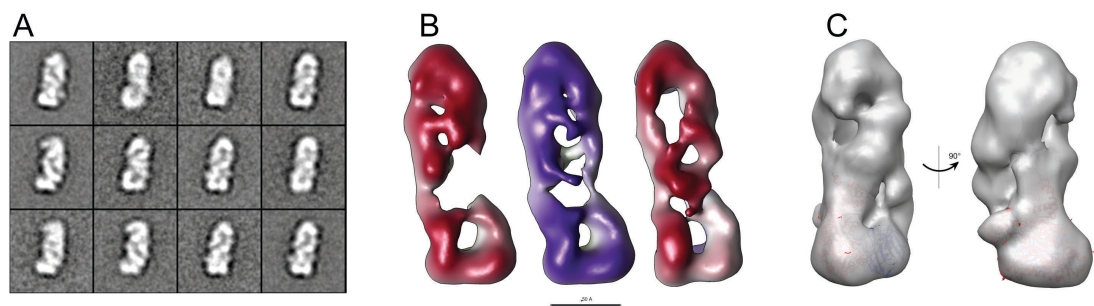

Figure 1. Initial reconstruction of the $\mathrm{Cmr}$ complex. (A) Class averages of reference-free aligned $\mathrm{Cmr}$ complex particles. (B) RCT models generated using similar particle classes from (A). (C) Threedimensional model of the $\mathrm{Cmr}$ complex from single-particle refinement. Scale bar represents $50 \AA$.
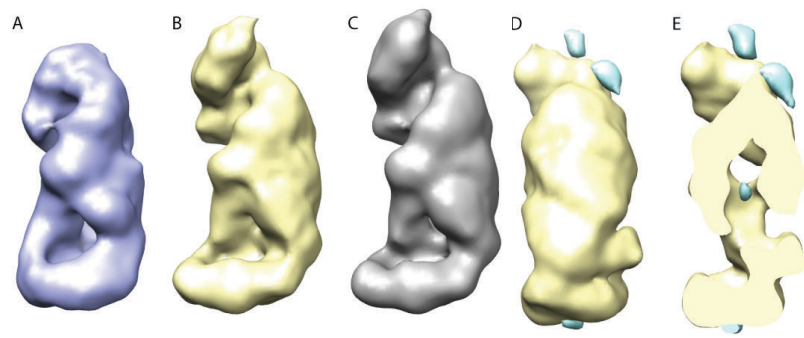

Figure 2. Identification of $\mathrm{Cmr} 1$ and target RNA by difference density mapping. (A) $\mathrm{Cmr2}-6+45$ guide, (B) Cmr1-6+45guide, and (C) Cmr1-6+45guide+37target negative stain structures. (D) Cmr1-6+45guide structure (yellow) together with a difference map (blue) between (B) and (C). The difference density is shown as $10 \sigma$. (E) The same view as (D) but clipped to highlight the difference density in the center of the complex. 45guide and 37target refer to the 45-nucleotide crRNA and 37-nucleotide target RNA, respectively.
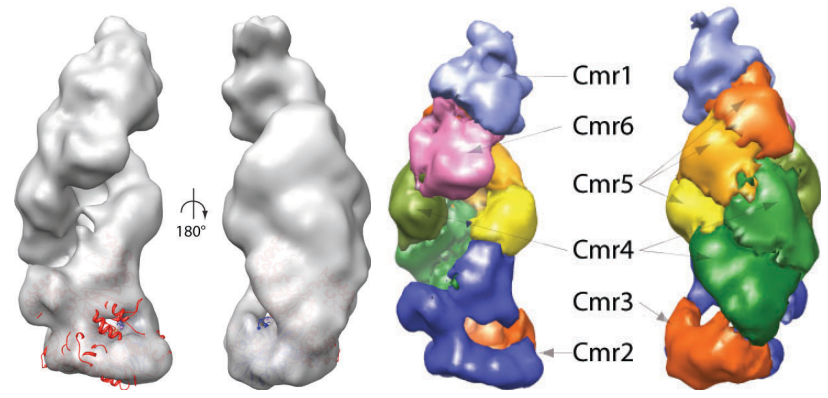

Figure 3. Cryo-EM reconstruction of the Cmr complex and subunit identification. A contour map is shown of Cmr1-6+45guide+37target with $\mathrm{Cmr} 2$ (red) and $\mathrm{Cmr} 3$ (blue) crystal structures (PDB ID: $4 \mathrm{H} 4 \mathrm{~K})$ docked into the map. Subunits were segmented by fitting Cmr homology models to the EM density and are identified by labels and arrows. 